\title{
Effect of the cap structure on pre-mRNA splicing in Xenopus oocyte nuclei
}

\author{
Kunio Inoue, Mutsuhito Ohno, Hiroshi Sakamoto, and Yoshiro Shimura \\ Department of Biophysics, Faculty of Science, Kyoto University, Kyoto 606, Japan
}

\begin{abstract}
The effect of the 5' cap structure on the splicing of precursor mRNAs was investigated after the RNAs were injected into Xenopus oocyte nuclei. The precursor mRNAs synthesized in vitro in a prokaryotic transcription system with a dinucleotide, ApppG, as a primer, were extremely stable when injected into the nuclei yet behaved like uncapped pre-mRNAs in the in vitro splicing reaction. The ApppG-primed precursor mRNAs served as a control (uncapped) in the injection experiments, and their splicing reactions were compared with those of their capped ( $\mathrm{m}^{7} \mathrm{GpppG}$-primed) counterparts. The capped precursors were spliced more efficiently than the uncapped precursors. Examination of splicing of the precursor mRNA that contained three exons and two introns within a single molecule has revealed that the cap structure exerts its effect primarily on the 5 'proximal intron. Thus, the cap structure not only stabilizes precursor mRNAs but also plays a positive role in the splicing of precursor mRNAs in cells.
\end{abstract}

[Key Words: Xenopus oocyte; microinjection; pre-mRNA splicing; cap structure]

Received September 9, 1988; revised version accepted June 13, 1989.

Since in vitro splicing systems were developed, considerable progress has been made in understanding the mechanism of nuclear precursor mRNA (premRNA) splicing (for review, see Green 1986; Padgett et al. 1986; Sharp 1987). A cap structure, $\mathrm{m}^{7} \mathrm{G}\left(5^{\prime}\right) \mathrm{ppp}\left(5^{\prime}\right) \mathrm{N}$, blocking the $5^{\prime}$ termini of many eukaryotic mRNAs has been shown to play an important role in pre-mRNA splicing (Krainer et al. 1984; Konarska et al. 1984; Edery and Sonenberg 1985; Ohno et al. 1987). Although the requirement for the cap appears to differ slightly between whole-cell and nuclear extracts (Konarska et al. 1984: Edery and Sonenberg 1985), the results obtained with the in vitro splicing reaction indicate that cap recognition is an important event in the early process of the splicing reaction. By use of pre-mRNAs that contain three exons and two introns, we have shown that when a pre-mRNA is capped, the upstream intron is spliced out more efficiently than the downstream intron because of the differential effect of the cap structure on the two introns. We have also shown that the cap structure exerts its effect primarily on the 5 '-proximal intron (Ohno et al. 1987). The aforementioned effect of the cap structure on pre-mRNA splicing was studied solely in in vitro systems, and it is not entirely clear whether the findings obtained in in vitro systems also apply to cells. It has been argued that one in vivo function of the cap is primarily to stabilize pre-mRNA molecules in the nucleus and that the in vivo role of the cap in splicing still remains to be clarified (Green 1986). One problem encountered in the in vivo study of cap function is that uncapped pre-mRNA is degraded rapidly upon injection into nuclei (Green et al. 1983), and consequently, comparative studies of the in vivo splicing reaction of capped
pre-mRNA and that of its uncapped counterpart have been difficult. We undertook a close examination of the in vivo role of the cap in pre-mRNA splicing by using pre-mRNAs that had a blocking nucleoside at the $5^{\prime}$ termini but that were not in the cap structure. When injected into the nuclei of Xenopus oocytes, these premRNAs were extremely stable but behaved like uncapped pre-mRNAs, thereby enabling us to separate the stability problem from the function of the cap structure. Here we show that the cap structure is required for efficient splicing of pre-mRNA in cells and that it enhances the excision of an intron proximal to the structure.

\section{Results}

In vitro synthesis of pre-mRNAs that have an A residue as the blocking nucleoside at their $5^{\prime}$ termini

To examine the effect of the $5^{\prime}$ cap structure on the splicing reaction in the nucleus of Xenopus oocytes, it is necessary to have as controls pre-mRNAs that behave like uncapped pre-mRNA but that are stable in the nucleus, because pre-mRNAs that contain triphosphate at the $5^{\prime}$ ends are known to be degraded rapidly in the nucleus. Pre-mRNAs that have an A residue as the blocking nucleoside instead of $\mathrm{m}^{7} \mathrm{G}$ at the $5^{\prime}$-terminal cap structure were expected to meet such requirements.

When linearized plasmids that contained portions of the $\delta$-crystallin gene were transcribed in the presence of $\mathrm{m}^{7} \mathrm{G}\left(5^{\prime}\right) \mathrm{ppp}\left(5^{\prime}\right) \mathrm{G}$ as a primer, $\delta E X$ pre-mRNAs that had $\mathrm{m}^{7} \mathrm{G}$ as the blocking nucleoside was produced, as described previously (Ohno et al. 1987; Sakamoto et al. 1987). Two kinds of $\delta E X$ pre-mRNAs were employed in 
the present study (Fig. 1). To prepare $\delta$ EX pre-mRNAs that had an A residue as the blocking nucleoside, we added a dinucleotide ApppG, instead of $\mathrm{m}^{7} \mathrm{GpppG}$ to the in vitro transcription reaction. The transcripts were labeled with $\left[\alpha^{-32} \mathrm{P}\right] \mathrm{CTP}$ and purified by polyacrylamide gel electrophoresis as described previously (Ohno et al. 1987). The priming activity of ApppG was almost comparable to that of $\mathrm{m}^{7} \mathrm{GpppG}$, although the former was consistently lower than the latter. To examine whether the ApppG dinucleotide indeed worked as the primer in the transcription system, thereby being incorporated into the transcript, the purified pre-mRNA was digested with RNase $T_{2}$, and the digests were separated by twodimensional thin-layer chromatography on a cellulose plate. It is worth noting that all the $\delta \mathrm{EX}$ pre-mRNAs have a sequence of $\left(5^{\prime}\right) \mathrm{GCAA}$ - at their $5^{\prime}$ termini if they are uncapped (Sakamoto et al. 1987). As shown in Figure $2 a$, two extra spots were detected together with the four canonical nucleotides (Ap, $\mathrm{Gp}, \mathrm{Cp}$, and $\mathrm{Up}$ ) in the chromatogram. One of them corresponded to the position of guanosine $5^{\prime}$-triphosphate $3^{\prime}$-monophosphate (pppGpl, as judged by its mobility on the chromatogram. This spot appeared to be derived from the unprimed transcript that had a triphosphate at the $5^{\prime}$ terminus. It is worth noting that $\sim 30 \%$ of the total transcripts synthesized with the ApppG primer were accounted for by the unprimed transcript. The occurrence of the other spot, designated $\mathrm{X}$, depended strictly on the addition of the ApppG primer to the transcription reaction (data not shown). Furthermore, the $T_{2}$ digests of the transcripts labeled with other $\left[\alpha^{-32}\right.$ P]NTPs (ATP, GTP, and UTP) did not yield this spot (data not shown). When the transcript was treated with alkaline phosphatase followed by digestion with RNase $T_{2}$, we found that the $X$ spot was resistant to alkaline phosphatase but the pppGp spot disappeared (data not shown). These results show that ApppG was actually used as a primer in the transcription reaction and that the first nucleotide linked to the primer was cytidylate. It is highly likely that the transcription start site with the ApppG primer was the same as that with the $\mathrm{m}^{7} \mathrm{GpppG}$ primer.

When ApppG was used as a primer, the blocking nucleoside could take three forms; that is, the blocking nucleoside could be A or G or both. To test which was
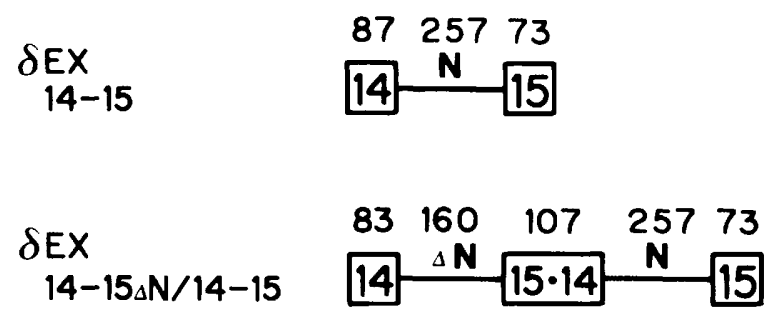

Figure 1. Schematic representation of the pre-mRNAs. Exon sequences are shown (boxes); the lines between the exons show intron sequences. The numbers within the boxes and the letters above the lines show specific exons and introns, respectively, in the $\delta$-crystallin gene (Ohno et al. 1985). The 5' terminus of each pre-mRNA is at the left of the figure. The length (in nucleotides) of the exons and introns is indicated above them.

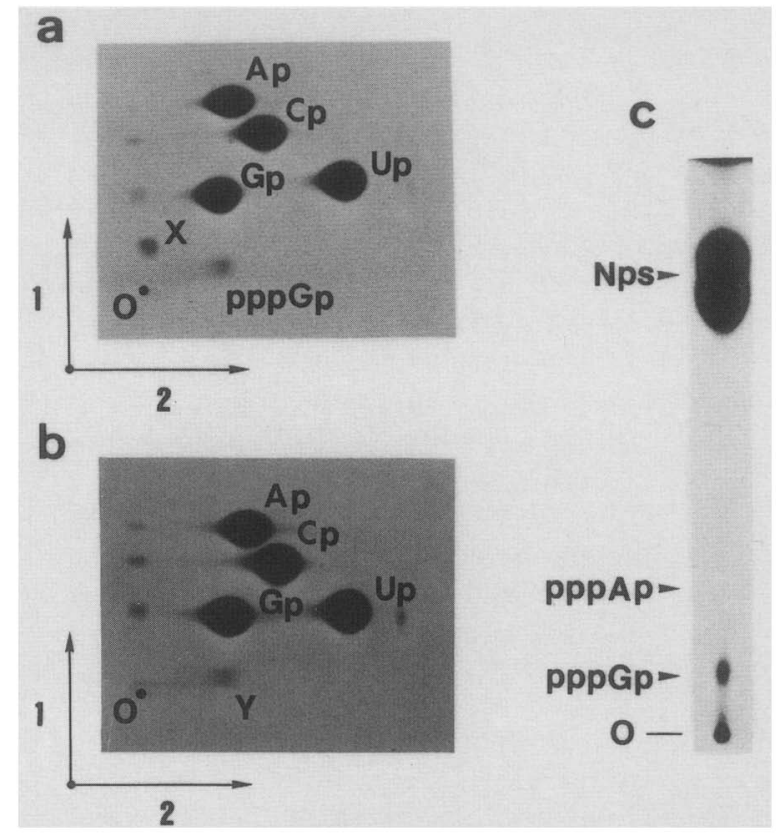

Figure 2. Analyses of the $5^{\prime}$-terminal structure of the premRNA primed with ApppG. $\delta$ EX14-15 pre-mRNA was prepared by primed synthesis by use of the ApppG dinucleotide in the presence of $|\alpha-32 \mathrm{P}| \mathrm{CTP}$ as described in Materials and methods. $(a)$ The pre-mRNA was digested completely with RNase $T_{2}$, and the digests were separated by two-dimensional thin-layer chromatography on a cellulose plate as described in Materials and methods. The oligonucleotide $\mathrm{X}$ derived from the $5^{\prime}$ terminus of the pre-mRNA is detailed in the text. $|b|$ The pre-mRNA was treated with alkaline phosphatase, decapped by periodate oxidation followed by $\beta$-elimination, and then digested with RNase $T_{2}$. The digests were separated as in $a$. The nucleotide $\mathrm{Y}$ is described in the text. $(c)$ The same $\mathrm{T}_{2}$ digests as in $b$ were chromatographed on a PEI cellulose plate. (Left) The positions of the nonradioactive nucleotides used as markers are indicated. In all the chromatograms, $O$ denotes the origin of chromatography.

actually the case, we had to employ the following cumbersome procedure because ApppGp and GpppAp markers were not available. The ApppG-primed $\delta \mathrm{EX14-}$ 15 pre-mRNA that had been purified by denaturing gel electrophoresis was treated with alkaline phosphatase to eliminate the $5^{\prime}$-terminal triphosphate of the contaminating unprimed pre-mRNA. To remove the blocking nucleoside, the RNA was oxidized with periodate at $0^{\circ} \mathrm{C}$, and then treated with aniline for $\beta$-elimination as described in Materials and methods. The RNA was washed by ethanol precipitation and then digested with RNase $T_{2}$. As shown in Figure $2 b$, the $T_{2}$ digests gave a spot (spot $\mathrm{Y}$ ) as well as canonical nucleotides in two-dimensional thin-layer chromatography. Further analysis of the $T_{2}$ digests by PEI cellulose chromatography revealed that the nucleotide corresponding to spot $Y$ comigrated with pppGp (Fig. 2c). It should be emphasized that we could not detect a spot that comigrated with pppAp. These results led us to conclude that the blocking nucleoside of the pre-mRNAs primed with ApppG was almost exclusively the A residue. 


\section{Effect of the cap structure on splicing of $\delta E X 14-15$ pre-mRNA in Xenopus oocyte nuclei}

We carried out the in vitro splicing reaction of $\delta \mathrm{EX} 14-$ 15 pre-mRNAs with the different $5^{\prime}$-terminal structures, namely, $\mathrm{m}^{7} \mathrm{G}$ or $\mathrm{A}$ as the blocking nucleoside. Various RNA bands produced in the reaction were characterized as described previously (Sakamoto et al. 1987). Although the $\mathrm{m}^{7} \mathrm{GpppG}$-primed pre-mRNA was spliced efficiently, the ApppG-primed pre-mRNA was spliced less efficiently (Fig. $3 \mathrm{~b}$ ). It is important to note that the profile of the splicing reaction of the ApppG-primed premRNA was similar to that of uncapped pre-mRNA in vitro (data not shown). In the following experiments, we used ApppG-primed pre-mRNAs instead of uncapped pre-mRNAs as a control for $\mathrm{m}^{7} \mathrm{GpppG}$-primed premRNAs (capped pre-mRNAs).

First, $\delta$ EX14-15 pre-mRNA synthesized with the $\mathrm{m}^{7} \mathrm{GpppG}$ primer was injected into the nuclei of Xenopus oocytes, and at various times after injection the oocytes were frozen quickly. RNA was extracted from the frozen oocytes as described in Materials and methods and then fractionated by polyacrylamide gel electrophoresis. As shown in Figure $3 a$, the spliced product was detected after 20 min together with RNA molecules that corresponded to the splicing intermediates detected in the in vitro reaction. The in vitro de-

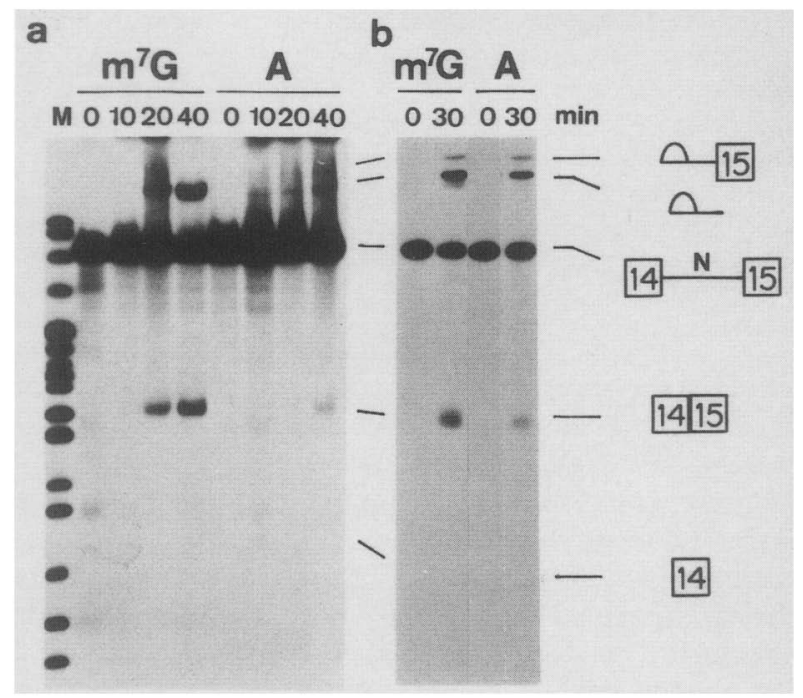

Figure 3. Splicing reactions of $\delta$ EX14-15 pre-mRNAs with different 5 '-terminal structures. $\delta$ EX14-15 pre-mRNAs were synthesized with $\mathrm{m}^{7} \mathrm{GpppG}$ or ApppG as a primer in the presence of $\left[\alpha-{ }^{32} \mathrm{P}\right] \mathrm{GTP}$. \{Lanes $m^{7} G \mid \mathrm{m}^{7} \mathrm{GpppG}$-primed pre-mRNA; (lanes A) ApppG-primed pre-mRNA. (a) The pre-mRNAs were injected into the nuclei of Xenopus oocytes, and total RNA was extracted at the time indicated at the top of each lane. $\langle b\rangle$ The pre-mRNAs were incubated with a HeLa cell nuclear extract at $30^{\circ} \mathrm{C}$ for $30 \mathrm{~min}$. The RNA products were electrophoresed on a $6 \%$ polyacrylamide gel containing $8 \mathrm{M}$ urea and autoradiographed for $20 \mathrm{hr}$. (Right) The structure of each RNA species is illustrated schematically. (Lane $M$ ) ${ }^{32}$ P-Labeled pBR322 DNA digested with HpaII used as size markers. branching assay of the lariat RNAs and the structural analysis of the RNA products showed that both the spliced sites and the branched site were the same as those encountered in the in vitro splicing reaction /data not shown|. About $40 \%$ of the input pre-mRNA was spliced after 40-min incubation.

Second, when $\delta$ EX14-15 pre-mRNA that was synthesized with the ApppG primer was injected into the Xenopus nuclei, and RNA products were analyzed as before, the efficiency of splicing was about a quarter of that observed with the capped pre-mRNA, although the ApppG-primed pre-mRNA was as stable as its $\mathrm{m}^{7} \mathrm{GpppG}$-primed counterpart in the Xenopus nuclei (Fig. 3a).

These results indicate clearly that the $5^{\prime}$ cap structure may play a positive role in the splicing reaction, and the stimulating effect is not merely the result of the stabilization of the pre-mRNA molecules in the nuclei. To confirm this notion further, we carried out a competition experiment, in which $\delta \mathrm{EX} 14-15$ pre-mRNA was coinjected with a competitor RNA that was synthesized in the SP6 transcription system by use of the $\mathrm{m}^{7} \mathrm{GpppG}$ or ApppG primer as described in Materials and methods. When the capped $\delta E X 14-15$ pre-mRNA and each competitor RNA were coinjected, the $\mathrm{m}^{7} \mathrm{GpppG}$-primed competitor RNA strongly inhibited the splicing reaction in the nuclei (Fig. 4, lanes 1-3), whereas the ApppG-

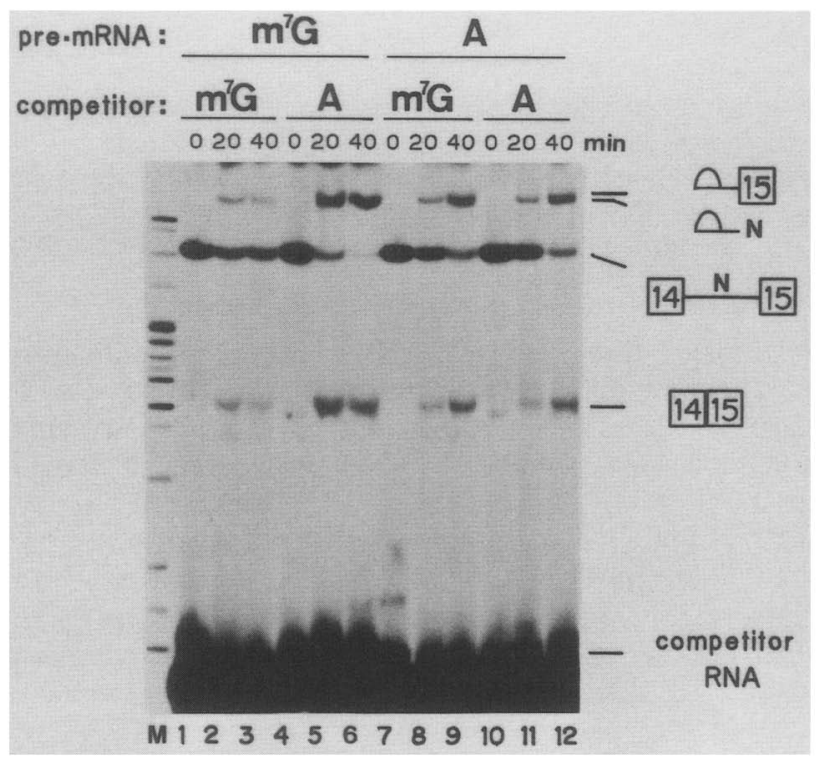

Figure 4. Splicing reactions of the $\delta \mathrm{EX} 14-15$ pre-mRNAs coinjected with competitor RNAs. The competitor RNAs were synthesized as described in Materials and methods. The $\delta$ EX $14-15$ pre-mRNAs primed with $\mathrm{m}^{7} \mathrm{GpppG}\left(m^{7} G\right.$, lanes $\left.1-6\right)$ or ApppG $(A$, lanes $7-12)$ was coinjected with about 70 -fold molar excess of the competitor RNA. (Lanes $1-3$ and 7-9) $\mathrm{m}^{7} \mathrm{GpppG}$-primed competitor RNA; (lanes 4-6 and 10-12) ApppG-primed competitor RNA. Total RNAs were extracted at the time indicated at the top of each lane and were electrophoresed on a $6 \%$ polyacrylamide gel containing $8 \mathrm{M}$ urea. (Lane $M$ ) ${ }^{32}$-Labeled pBR322 DNA digested with HpaII used as size markers. 
primed competitor RNA had virtually no effect (Fig. 4, lanes 4-6). On the other hand, when the ApppG-primed pre-mRNA and each competitor RNA were coinjected, both competitor RNAs did not affect the splicing reaction of the ApppG-primed pre-mRNA (Fig. 4, lanes 7-12). Therefore, only the capped competitor RNA abolished the stimulating effect of the cap structure of premRNA in the splicing reaction.

Effect of the $5^{\prime}$ cap structure on the splicing reaction of pre-mRNAs containing two introns in Xenopus oocyte nuclei

As mentioned earlier, the salient feature of the cap effect on the in vitro splicing reaction is that the cap structure enhances splicing of only the 5'-proximal intron of pre-
mRNA that contain two introns. An attempt was made to examine whether this was also the case in cells. We constructed the plasmid $\delta$ EX14-15 $\Delta \mathrm{N} / 14-15$, in which a shortened version (160 bp, intron $\Delta N$ ) of intron $N$ and the intact intron $\mathrm{N}(257 \mathrm{bp})$ were present with three exons (Fig. 1). The middle exon had chimeric sequences of exons 15 and 14. The natural sequences were preserved in all the splice sites and the branch sites. $\delta$ EX14-15 $\Delta \mathrm{N} / 14-15$ pre-mRNA was prepared from this plasmid as described in Materials and methods. Splicing of $\delta \mathrm{EX} 14-15 \Delta \mathrm{N} / 14-15$ pre-mRNAs with different $5^{\prime}-$ terminal structures was examined both in vitro (Fig. 5a) and in oocyte nuclei (Fig. 5b).

As shown in Figure $5 a$, when the $\mathrm{m}^{7} \mathrm{GpppG}$-primed $\delta$ EX14-15 $\Delta \mathrm{N} / 14-15$ pre-mRNA was incubated with a HeLa nuclear extract, the splicing reaction occurred effi-

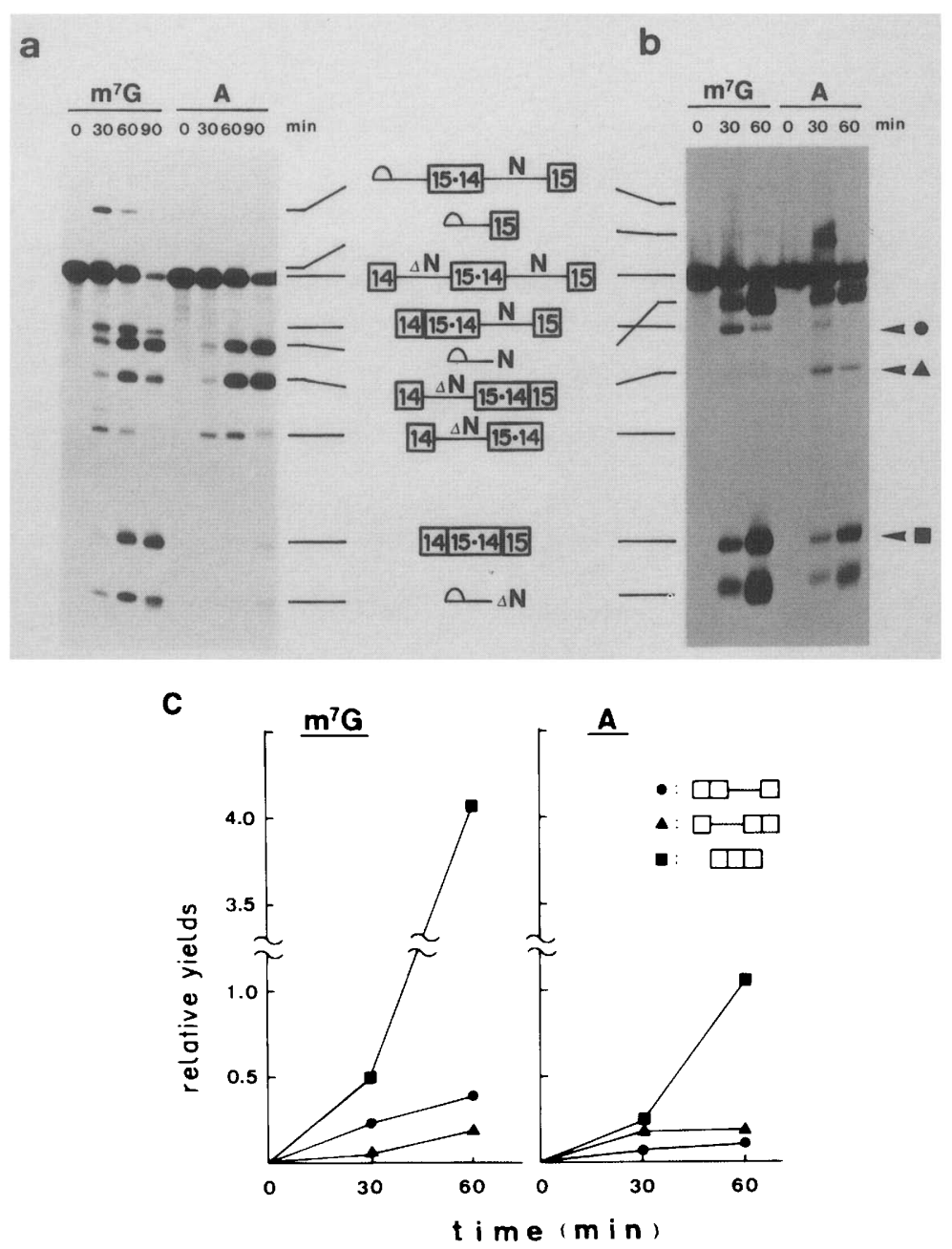

Figure 5. Kinetics of the splicing reactions of $\delta \mathrm{EX} 14-15 \Delta \mathrm{N} / 14-15$ pre-mRNAs with different $5^{\prime}$-terminal structures. $\delta \mathrm{EX} 14-15 \Delta \mathrm{N} /$ 14-15 pre-mRNAs primed with $\mathrm{m}^{7} \mathrm{GpppG}\left(\mathrm{m}^{7} \mathrm{G}\right)$ or ApppG $(A)$ were incubated with a HeLa cell nuclear extract $(a)$ or injected into oocytes $(b)$. Total RNAs were extracted at the time indicated at the top of each lane, and the products were electrophoresed on a $5 \%$ polyacrylamide gel containing $8 \mathrm{M}$ urea. The autoradiogram shown in $a$ was obtained by exposure for $12 \mathrm{hr}$, whereas that shown in $b$ was obtained by exposure for $30 \mathrm{hr}$. The gel pieces in $b$ were cut out, and their radioactivities were measured by Cerenkov counting. (c) The molar ratios of the three RNA species to the pre-mRNAs remaining unspliced were calculated and plotted as the relative yields. $(\bullet)$ Molecule in which only the upstream intron $(\Delta N)$ was excised; $(\Delta)$ molecule in which only the downstream intron $(N)$ was excised; (ø) final spliced product. 
ciently, generating the final spliced product in which three exons were ligated in order. There were two kinds of RNA molecules in which only one of the two introns was spliced out. When the capped pre-mRNA was used, the inolecule in which only the upstream intron (intron $\Delta N$ ) was excised (designated RNA U) was produced appreciably after $60 \mathrm{~min}$ but decreased gradually afterwards. The molecule in which only the downstream intron (intron N) was removed (designated RNA D) appeared somewhat later. The kinetics of these reactions are almost consistent with those observed previously with other pre-mRNA species that contain two introns (Ohno et al. 1987). On the other hand, when the ApppGprimed pre-mRNA was employed, the final spliced product was undetectable and the amount of RNA U was extremely reduced, whereas the production of RNA $\mathrm{D}$ increased considerably. These results obtained with the ApppG-primed pre-mRNA are essentially the same as those observed previously with uncapped (unprimed) pre-mRNAs (Ohne et al. 1987). Thus, the ApppG-primed substrate appears to behave like its unprimed counterpart in the in vitro splicing reaction, although it is as stable as the capped pre-mRNA in HeLa cell nuclear extracts.

The results obtained from the injection experiment were consistent with those obtained from the in vitro experiments, although the gel electrophoretic mobility of lariat RNA molecules was slightly different because of the different electrophoretic conditions (Fig. 5b). It should be pointed out that intermediate molecules, except for the intron lariat, were unstable and difficult to detect in the injected nuclei. Accordingly, the autoradiogram shown in Figure $5 \mathrm{~b}$ was obtained by exposure for prolonged time $(30 \mathrm{hr})$ compared to those shown in Figures 3 and $5 \mathrm{a}(12 \mathrm{hr})$. Although precise quantification of the spliced products in the Xenopus nuclei was difficult because of their instability, the RNA bands in Figure $5 \mathrm{~b}$ were recovered, like those in Figure $5 \mathrm{a}$, and quantitated. The molar ratios of the three RNA species, RNA U, RNA D, and the final spliced product, to the pre-mRNAs remaining unspliced in the oocytes were plotted (Fig. 5c). When the capped $\delta$ EX14-15 $\Delta \mathrm{N} / 14-15$ precursor was injected, RNA $U$ and the final products were generated efficiently, whereas RNA D was barely detectable. However, when the ApppG-primed premRNA was used, splicing of the upstream intron was inhibited remarkably and the amount of the final product was reduced to about a quarter of that obtained with its capped counterpart, whereas the production of RNA D increased slightly. It appears, therefore, that when the pre-mRNA is capped, the upstream intron is spliced more efficiently than the downstream intron, although the differential effect of the cap structure appears to be slightly less dramatic in the injection experiment as compared to the effect in the in vitro reaction. To eliminate the possibility that these results were caused by a difference in the intrinsic efficiency of the splicing reactions of the two introns, the following experiments were carried out.

When the two splicing reactions that included the ex- cising of the upstream and the downstream introns were compared by use of pre-mRNAs that contained single introns $(\delta \mathrm{EX} 14-15$ and $\delta \mathrm{EX} 14-15 \Delta \mathrm{N}$; see Materials and methods), the splicing efficiency of $\delta E X 14-15$ was $1.2-1.8$ times higher than that of $\delta \mathrm{EX} 14-15 \Delta \mathrm{N}$ in the in vitro reaction system with $\mathrm{HeLa}$ cell nuclear extracts and also in the microinjection system (data not shown). Furthermore, when splicing of two introns was tested in the oocyte nuclei by use of $\delta E X 14-15 / 14-15 \Delta \mathrm{N}$ premRNA, in which the relative order of the two introns of $\delta E X 14-15 \Delta N / 14-15$ was reversed, the results were consistent with those of $\delta \mathrm{EX} 14-15 \Delta \mathrm{N} / 14-15$, that is, the upstream intron $(\mathrm{N})$ was spliced out more efficiently than the downstream intron $(\Delta N)$ if the pre-mRNA was capped (data not shown). Thus, efficient splicing of the upstream intron of capped $\delta \mathrm{EX} 14-15 \Delta \mathrm{N} / 14-15$ premRNA in the oocyte nuclei as opposed to the downstream intron did not result from a difference in the intrinsic efficiency of the splicing of the two introns.

\section{Discussion}

We have examined the effect of the cap structure on splicing in cells and have shown that the structure has a positive role in the splicing reaction. Furthermore, by use of a pre-mRNA that contains two introns, we demonstrated that, in cells, the cap structure exerts a stimulating effect on the excision of the intron proximal to the structure. These results are totally consistent with our previous findings with the in vitro system (Ohno et al. 1987).

Previous workers have shown that an uncapped premRNA is degraded immediately when injected into the oocyte nuclei (Green et al. 1983). The instability of uncapped pre-mRNAs upon injection into the nuclei has been a major drawback of investigations on the effect of the cap structure on pre-mRNA splicing in vivo. To circumvent this impasse, we employed pre-mRNAs that were synthesized in vitro using a dinucleotide, ApppG, as a primer. In our system, the pre-mRNA molecules thus synthesized had almost exclusively adenylate (but not guanylatel as the blocking nucleoside, because we could not detect pppAp upon the periodate oxidation coupled with $\beta$-elimination of the $5^{\prime}$ terminus of the pre-mRNA. The ApppG-primed pre-mRNA molecules were extremely stable both in vitro and in nuclei as compared with their uncapped counterparts. For example, in the oocyte nuclei, $60-70 \%$ of the uncapped RNA and $20 \%$ of the ApppG-primed RNA introduced were degraded after a 40-min incubation (data not shown). This value $(20 \%)$ is an underestimate of the stability of the ApppG-primed RNA for the following reason. We estimate that about $30 \%$ of the population of the ApppG-primed pre-mRNA actually represents unprimed (uncapped) pre-mRNA, because the ratio of the pppGp spot to the ApppGp spot (X) obtained by RNase $\mathrm{T}_{2}$ digestion of the ApppG-primed pre-mRNA was about 3:7 as shown in Figure 2a. Thus, the degradation of the ApppG-primed pre-mRNA appears to result predominantly from the contaminating uncapped RNA. 
The ApppG-primed pre-mRNA behaved like the uncapped pre-mRNA in the in vitro splicing reaction, although the stability of the ApppG-primed pre-mRNA was comparable to that of the capped ( $\mathrm{m}^{7} \mathrm{GpppG}$-primed) pre-mRNA. Accordingly, the ApppG-primed premRNAs enabled us to make a comparative analysis of the splicing reactions of the capped and uncapped premRNAs in cells.

We demonstrated that the cap structure plays a positive role in the splicing reaction in the oocyte nuclei. This was shown when, in the nuclei, splicing of the capped pre-mRNA was inhibited by coinjection with a 70-fold molar excess of the $\mathrm{m}^{7} \mathrm{GpppG}$-primed (capped) competitor RNA. This inhibition is, in all likelihood, the result of competition between the cap structure of the competitor RNA and the cap structure of the premRNA but not of that between the cap structures of the competitor RNA and snRNAs, because this competitor has no effect on the splicing reaction of the ApppGprimed pre-mRNA in oocyte nuclei. In the case of the capped $\delta$ EX14-15 $\Delta \mathrm{N} / 14-15$ pre-mRNA containing two introns, the production of the final spliced product was inhibited also by coinjection with the capped competitor RNA (data not shown). In contrast, the in vitro splicing reaction of the pre-mRNA was not inhibited by coincubation with the same amount (70-fold molar excess) of the capped competitor RNA. The mechanism underlying the differential sensitivity of the two splicing systems to the capped competitor RNA is not understood. It is noteworthy in this connection that cap analog, $\mathrm{m}^{7} \mathrm{GTP}$, did not inhibit the splicing reaction in the oocyte nuclei, when coinjected with the capped premRNA, although the nucleotide inhibited the in vitro splicing reactions if HeLa cell nuclear extracts were preincubated with this cap analog before the reaction. The insensitivity of the splicing reaction in the oocyte nuclei to cap analogs remains to be elucidated.

As shown previously (Ohno et al. 1987), the splicing reaction occurs accurately with uncapped pre-mRNAs. In the case of $\beta$-globin pre-mRNA, $30-40 \%$ of the uncapped substrate was spliced for $4 \mathrm{hr}$ in vitro (Krainer et al. 1984). Our data show that about $10 \%$ of the ApppGprimed $\delta E X$ pre-mRNAs are spliced for $40 \mathrm{~min}$ in vitro and also in Xenopus oocyte. Although the splicing efficiencies of these different pre-mRNAs were somewhat variable when they were uncapped, their splicing efficiency was always enhanced about threefold if they were capped.

The present data obtained from the injection experiments show that the spliced products of the capped premRNAs are degraded more rapidly than those of the ApppG-primed pre-mRNAs in nuclei. For example, we estimate that, when the $\delta$ EX14-15 pre-mRNA was injected and incubated for $60 \mathrm{~min}$ in oocyte nuclei, more than $70 \%$ of the final spliced product was degraded if the pre-mRNA was capped, whereas only $20-30 \%$ of the ApppG-primed counterpart was degraded /data not shown). Thus, when the capped SEX14-15 pre-mRNA was coinjected with the competitor RNAs, the amounts of the spliced products obtained after 40-min incubation
(Fig. 5, lanes 3 and 6) were less than those obtained after 20-min incubation (Fig. 5, lanes 2 and 5). It is possible that some degradation machinery, including decapping activity, may exist in cells and degrade these RNAs. However, the machinery cannot degrade the ApppGprimed RNAs, since such molecules never exist in nature.

When the ApppG-primed pre-mRNA containing two introns $(\delta E X 14-15 \Delta N / 14-15)$ was injected into oocyte nuclei, the production of the final spliced molecule was reduced to about a quarter of that observed with the capped counterpart. It is possible that splicing proceeds via two pathways: one via RNA $U$ in which only the upstream intron is removed, and the other via RNA D in which only the downstream intron is excised. In the splicing reaction of the capped pre-mRNA in vivo as well as in vitro, the final spliced product seems to be produced mainly via RNA U on the basis of the kinetics of their appearance. This is in contrast to the case obtained with the uncapped counterpart. Although we cannot rule out the possibility that some marginal amount of the final spliced product is produced via RNA $\mathrm{D}$, the majority of the product appears to be derived from RNA U. These results are consistent with our previous in vitro experiments showing that the cap structure exerts its enhancing effect primarily on the 5 '-proximal intron (Ohno et al. 1987). As a consequence of the differential effect of the cap structure on the two introns, it appears as if the overall splicing reaction proceeds in a processive manner from the $5^{\prime}$ side of the pre-mRNA. However, this does not mean that the 5 '-proximal intron of multiple intron pre-mRNAs is always excised first. Several cases are known where the $5^{\prime}$-proximal intron of multiple intron pre-mRNA is not necessarily excised preferentially (Gattoni et al. 1986; Christofori et al. 1987; Lang and Spritz 1987). It has been demonstrated also that two spliceosomes can form simultaneously and independently on synthetic double-intron pre-mRNAs (Christofori et al. 1987). The simultaneous formation of two spliceosomes on a double-intron pre-mRNA as judged by sucrose-gradient centrifugation does not necessarily imply that splicing of the two introns proceeds simultaneously; thereby no orderliness exists in splicing of a double-intron pre-mRNA. It is highly likely that each intron has its own intrinsic splicing efficiency, which is possibly determined by numerous factors, including sequence of both splice sites, secondary structure of the pre-mRNA, exon sequences, exon length, etc. Thus, each intron is considered to be spliced out with different efficiency. If two introns, having similar intrinsic splicing efficiency, reside within a single premRNA molecule, the $5^{\prime}$-proximal intron is excised kinetically faster than the other as a consequence of the differential effect of the cap structure. On the other hand, if the intrinsic splicing efficiency of the upstream intron is much lower than that of the downstream intron, there may be a case where the latter intron is excised faster even in the presence of the cap structure. In previous works in which it was claimed that no preferential order of excision of the two identical introns 
within a single pre-mRNA was observed (Christofori et al. 1987), real intermediates such as RNAs $U$ and D were not quantitated and arguments were based mainly on the time course of appearance of the products such as $5^{\prime}$ exons or lariats. Moreover, the effect of the cap structure was not examined. It would be of interest to examine the effect of the cap structure on splicing of the pre-mRNA used by Christofori et al. (1987).

It has been proposed that the effect of the cap structure on the splicing reaction is mediated by a factor or factors that specifically recognize the structure of the pre-mRNAs. Attempts are being made in our laboratory to identify such factors in HeLa cell nuclear extracts. It is highly likely that similar factors are also present in the Xenopus nuclei and participate in pre-mRNA splicing.

\section{Materials and methods}

\section{Chemicals and enzymes}

32P-Labeled nucleotides were obtained from Amersham. Nonradioactive nucleotides including $\mathrm{m}^{7} \mathrm{GpppG}$ and ApppG were obtained from Pharmacia. RNA polymerase was purified to near homogeneity from Escherichia coli A19 as described previously (Nakajima et al. 1982). RNase $\mathrm{T}_{2}$ was obtained from Sankyo. All other enzymes were purchased from Takara Shuzo Company.

\section{Preparation of pre-mRNAs}

The plasmid p $\delta \mathrm{EX} 14-15$ containing two exons (exons 14 and 15 ) and one intron (intron N) of the $\delta$-crystallin gene was described previously (Sakamoto et al. 1987). A portion of nonessential intron sequence spanning the SacI and StyI restriction sites was deleted from this plasmid. The shortened plasmid was designated p $\delta \mathrm{EX} 14-15 \Delta \mathrm{N}$. A HindIII-KpnI fragment containing both the two exons and the shortened intron (intron

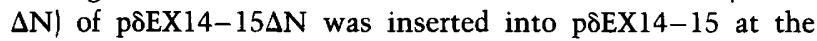
unique HindIII site which resides immediately upstream of exon 14. The resulting plasmid p $\delta$ EX14-15 $\Delta \mathrm{N} / 14-15$ consisted of the three exons and the two introns. These plasmids were digested with $\mathrm{XmnI}$. With these fragments as templates, pre-mRNAs were synthesized with $E$. coli RNA polymerase in the presence of $\left[\alpha^{-32}\right.$ P]GTP or $\left[\alpha^{-32}\right.$ P]CTP (Nakajima et al. 1982). The dinucleotide, $\mathrm{m}^{7} \mathrm{GpppG}$ or ApppG ( $\left.1 \mathrm{mM}\right)$, was added as primer in the transcription reaction. The transcripts were purified by electrophoresis on a $5 \%$ polyacrylamide- $8 \mathrm{M}$ urea gel as described previously (Ohno et al. 1987).

\section{Analysis of the 5'-terminal structure of pre-mRNA primed with ApppG}

The blocking nucleosides of pre-mRNAs were removed by periodate oxidation $\left[0.25 \mathrm{~mm} \mathrm{NaIO} 4_{4}-0.15 \mathrm{M} \mathrm{NaOAc}(\mathrm{pH} 5.3)\right.$ at $0^{\circ} \mathrm{C}$ for $1 \mathrm{hr}$ ], which was followed by $\beta$-elimination with $0.25 \mathrm{M}$ aniline $(\mathrm{pH} 5.3)$ at $30^{\circ} \mathrm{C}$ for $50 \mathrm{~min}$. The RNAs were washed by ethanol precipitation and then digested with RNase $T_{2}$. RNase $\mathrm{T}_{2}$ digestion and two-dimensional thin-layer chromatography on cellulose plates were carried out as described previously (Sakamoto et al. 1987). Chromatography on polyethyleneimine (PEI)-cellulose plates was performed in $0.85 \mathrm{M} \mathrm{KH}_{2} \mathrm{PO}_{4}(\mathrm{pH} 3.4)$ (Nakajima et al. 1982).

\section{In vitro splicing reaction}

A HeLa cell nuclear extract was prepared as described previously (Dignam et al. 1983). The splicing reactions and the subsequent fractionation of the reaction products were carried out as described previously (Sakamoto et al. 1987). Usually 10 fmoles of ${ }^{32} \mathrm{P}$-labeled pre-mRNA $\left(2-3 \times 10^{4} \mathrm{cpm}\right)$ were added per incubation.

\section{Microinjection}

Microinjections were performed as described previously /Green et al. 1983). A 25-nl solution of RNA (2-3 fmoles of premRNA) was injected into each Xenopus oocyte nucleus. A group of 20 oocytes was injected for each experiment and incubated at $20^{\circ} \mathrm{C}$ in a modified Barth solution (Gurdon 1976). At various times after injection, the oocytes were frozen rapidly in liquid nitrogen. RNA was extracted from the frozen oocytes by homogenization in GTC solution [4 $\mathrm{M}$ guanidine thiocyanate, $18 \mathrm{~mm}$ sodium citrate, $0.36 \%$ sarcosyl, $200 \mathrm{~mm}$ 2-mercaptoethanol (pH 7.0), phenol-chloroform extraction, and isopropanol precipitation.

Competitor RNAs were synthesized as follows; pSP64 DNA linearized with EcoRI was transcribed in vitro with SP6 RNA polymerase in the presence of $\left[\alpha^{-32} \mathrm{P}\right] \mathrm{GTP}$ and the primer dinucleotide, $\mathrm{m}^{7} \mathrm{GpppG}$ or ApppG (1 $\left.\mathrm{mm}\right)$, as described previously (Melton et al. 1984). The transcripts were purified by denaturing polyacrylamide gel electrophoresis.

\section{Acknowledgments}

We are grateful to Dr. H. Kubota of the Department of Zoology of Kyoto University for teaching us the microinjection techniques. We thank Dr. K. Mizumoto of the Institute of Medical Science of the University of Tokyo for valuable advice on the analysis of the cap structure. We also thank Karin Knisely for proofreading the manuscript. This work was supported by a Grant-in-Aid for Scientific Research (grant no. 62065009) from the Ministry of Education, Science, and Culture of Japan.

\section{References}

Christofori, G., D. Frendewey, and W. Keller. 1987. Two spliceosomes can form simultaneously and independently on synthetic double-intron messenger RNA precursors. $E M B O$ I. 6: 1747-1755.

Dignam, J.D., R.M. Lebovitz, and R.G. Roeder. 1983. Accurate transcription initiation by RNA polymerase II in a soluble extract from isolated mammalian nuclei. Nucleic Acids Res. 11: 1475-1489.

Edery, I. and N. Sonenberg. 1985. Cap-dependent RNA splicing in a HeLa nuclear extract. Proc. Natl. Acad. Sci. 82: 75907594.

Gattoni, R., P. Keohavong, and J. Stevenin. 1986. Splicing of the E2A premessenger RNA of adenovirus serotype 2. I. Mol. Biol. 187: 379-397.

Green, M.R. 1986. Pre-mRNA splicing. Annu. Rev. Genet. 20: $671-708$.

Green, M.R., T. Maniatis, and D.A. Melton. 1983. Human $\beta$ globin pre-mRNA synthesized in vitro is accurately spliced in Xenopus oocyte nuclei. Cell 32: 681-694.

Gurdon, J.B. 1976. Injection nuclei in frog oocyte: Fate, enlargement, and chromatin dispersal. J. Embryol. Exp. Morphol. 36: 523-540.

Konarska, M.M., R.A. Padgett, and P.A. Sharp. 1984. Recogni- 
tion of cap structure in splicing in vitro of mRNA precursors. Cell 38: 731-736.

Krainer, A.R., T. Maniatis, B. Ruskin, and M.R. Green. 1984. Normal and mutant human $\beta$-globin pre-mRNAs are faithfully and efficiently spliced in vitro. Cell 36: 993-1005.

Lang, K.M. and R.A. Spritz. 1987. In vitro splicing pathways of pre-mRNAs containing multiple intervening sequences. Mol. Cell. Biol. 7: 3428-3437.

Melton, D.A., P.A. Krieg, M.R. Rebagliati, T. Maniatis, K. Zinn, and M.R. Green. 1984. Efficient in vitro synthesis of biologically active RNA and RNA hybridization probes from plasmids containing a bacteriophage SP6 promoter. Nucleic Acids Res. 12: 7035-7056.

Nakajima, N., H. Ozeki, and Y. Shimura. 1982. In vitro transcription of the supB-E tRNA operon of Escherichia coli. J. Biol. Chem. 257: 11113-11120.

Ohno, M., H. Sakamoto, K. Yasuda, T.S. Okada, and Y. Shimura. 1985. Nucleotide sequence of a chicken $\delta$-crystallin gene. Nucleic Acids Res. 13: 1593-1606.

Ohno, M., H. Sakamoto, and Y. Shimura. 1987. Preferential excision of the 5 '-proximal intron from mRNA precursors with two introns as mediated by the cap structure. Proc. Natl. Acad. Sci. 84: 5187-5191.

Padgett, R.A., P.J. Grabowski, M.M. Konarska, and P.A. Sharp. 1986. Splicing of messenger RNA precursors. Annu. Rev. Biochem. 55: 1119-1150.

Sakamoto, H., M. Ohno, K. Yasuda, K. Mizumoto, and Y. Shimura. 1987. In vitro splicing of a chicken $\delta$-crystallin premRNA in a mammalian nuclear extract. $J$. Biochem. 102: $1289-1301$.

Sharp, P.A. 1987. Splicing of messenger RNA precursors. Science 235: 766-771. 


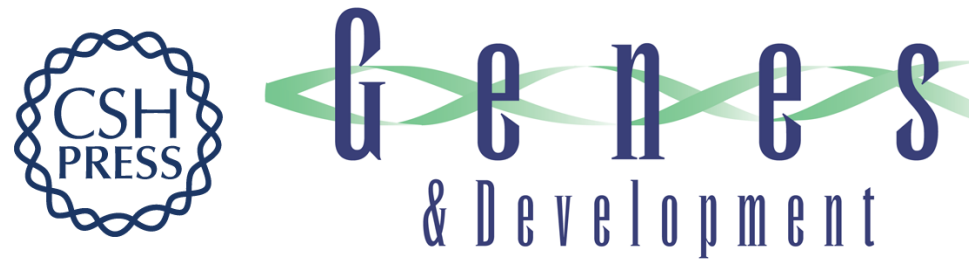

\section{Effect of the cap structure on pre-mRNA splicing in Xenopus oocyte nuclei.}

K Inoue, M Ohno, H Sakamoto, et al.

Genes Dev. 1989, 3:

Access the most recent version at doi:10.1101/gad.3.9.1472

References This article cites 17 articles, 5 of which can be accessed free at:

http://genesdev.cshlp.org/content/3/9/1472.full.html\#ref-list-1

License

Email Alerting

Service

Receive free email alerts when new articles cite this article - sign up in the box at the top right corner of the article or click here.

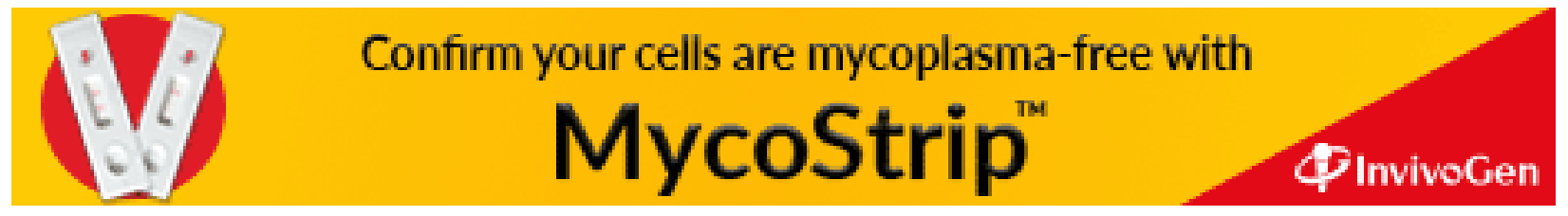

